\title{
A Simplified Synchronous Pulse-Width Modulation Method for Three-level Inverter of High-speed Train
}

\author{
Fei Lin ${ }^{1}$, Qiaona Lian ${ }^{1}$, Jialiang Yuan ${ }^{1}$, Zhongping Yang $^{1}$, Zhiqiang Zhang ${ }^{2}$, Yue \\ $\mathrm{Xu}^{2}$ and Jinghai Jiao \\ ${ }^{1}$ School of Electrical Engineering, Beijing Jiaotong University, No.3 \\ ShangyuanCun, Beijing, 100044 \\ flin@bjtu.edu.cn (F.L.); 13121421@bjtu.edu.cn (Q.L.); \\ 13121422@bjtu.edu.cn(J.Y.) zhpyang@bjtu.edu.cn (Z.Y.) \\ ${ }^{2}$ CSR Qingdao Sifang Co., Ltd., Qingdao, 266000 \\ zzqiang@cqsf.com(Z.Z.); xuyue@cqsf.com(Y.X.);sf-jiaojinghai@cqsf.com(J.J.);
}

\begin{abstract}
The synchronous Pulse-Width Modulation (PWM) is quite complex for three-level neutral point-clamped (NPC) inverter. A simple central $60^{\circ}$ synchronous modulation method is presented in this paper for three-level NPC inverter used in the multi-mode $P W M$ strategy. For the fundamental voltage command, the switching angle could be real-time calculated based on this modulation method with different carrier frequency ratios. The switching strategy between these modes is discussed for multi-mode PWM. According to this method, a simulation model of the drive system with three-level inverter and induction motor is built. The simulation results at asynchronous modulation, central $60^{\circ}$ synchronous modulation and square wave area, show that the output voltage values can meet the requirements with a good symmetry even in the case of low carrier frequency ratio. The smooth switching among different modes is also accomplished with low impulse current.
\end{abstract}

Keywords: AC drive; neutral-point clamped three-level inverter; multi-mode PWM; synchronous modulation; central $60^{\circ}$ modulation;

\section{Introduction}

The diode-clamped three-level inverters have certain advantages over conventional two-level inverters [1]. Each switching device of the three-level inverters withstands half of the DC link voltage, which can reduce the breakdown voltage of the devices. On the other hand, the output phase voltage has three levels, which improves current total harmonic distortion (THD) compared to a two-level inverter. Because of these features, three-level inverters have been widely used recently [2].

To use the asynchronous modulation in the whole range of speed will make the control strategy to be easier and liable to be realized for the high-power AC traction drive system. When the high-speed train is in the traction mode and the speed is rising up to the range of high speed, the frequency of the induction motor will increase to one or two hundred Hertz. While the carrier frequency of the asynchronous modulation is maintained constant at the same time, which will cause the carrier-to-modulating frequency ratio to go down. This gives rise to the impact of output waveform without asymmetry, and the harmonic effect tends to be worse [3-5]. On the other hand, the sampling number will go down, as the regular-sampled asynchronous modulation frequency ratio decreases. This will cause the amplitude of output fundamental voltage to be lower than the modulating wave. If the asynchronous modulation continues to be used in this case, the sampling frequency should 
be increased. This will cause large switching losses and also go against energy saving for high-power three-level inverter [6-8].

In order to reduce the switching losses and save energy, the multi-mode PWM is widely used presently, which can also reduce the lower order harmonics. The multi-mode PWM uses asynchronous mode at low fundamental frequency, synchronous mode at middle fundamental frequency, and square wave mode near the base frequency switching. The switching frequency can be set under one thousand Hertz when the asynchronous is used only in the low speed range. As the speed increases, transition occurs to the synchronous modulation. Generally, the highest switching frequency of the high-power three-level inverters is limited to hundreds of Hertz in high-speed train. In addition to this, the three-level inverters need to be operated in square-wave mode when the fundamental frequency reaches the base frequency of the traction motor, where the maximum voltage will be obtained.

In the synchronous modulation mode stage, the output fundamental voltage of the inverters controlled by the conventional regular-sampled PWM can't reach the voltage command, which makes it difficult to transform the modulation mode to square wave [9]. Hence, several synchronous optimal PWM strategies are developed, such as selected harmonic elimination PWM (SHEPWM) [10-14], minimum ripple current PWM [15], and so on. However, for all these synchronous optimal PWM, the switching angles are often calculated offline and determined using values stored in look-up tables [16]. An approximate method has been proposed in paper [17, 18], and the offline switching angles can be approximated using regular-sampled techniques. This method has advantages over other switching techniques, but it is only suitable to apply this method in a certain range of the fundamental frequency. A stator current trajectory tracking approach has been proposed in paper $[19,20]$. With this method the offline switching angles can be used during a dynamic process. On the other hand, it requires a fast identification of the inductance parameters of the drive motor. A modified method, stator flux trajectory tracking control, which can avoid such complication, is then presented in paper [21-23]. But both the two methods are difficult to be implemented.

This paper applies central $60^{\circ}$ synchronous modulation in three-level inverters, which has been proposed and well applied in two-level inverters. The switching angles can be calculated online and the output voltage values can meet the requirements with a good symmetry even in the case of low carrier frequency ratio. And, it is easy to transform the modulation mode to square wave.

\section{Central $60^{\circ}$ Synchronous Modulation for Three-level Inverters}

\subsection{Multi-mode PWM for Three-level Inverters}

Figure 1 shows the circuit diagram of a three-level neutral point-clamped (NPC) inverter. The DC source of voltage $U_{\mathrm{d}}$ is divided equally by two DC-link capacitors. The output phase voltage of inverter can obtain three levels $+u_{\mathrm{d}} / 2,0$, or $-u_{\mathrm{d}} / 2$.

Figure 2 shows the multi-mode modulation PWM strategy, which is often applied in high-power inverters. As indicated in the Figure 2, in the asynchronous modulation region, the carrier frequency $f_{\mathrm{c}}$ is maintained constant and the ratio $N$ is relatively high, where $N=f_{\mathrm{c}} / f_{\mathrm{s}}, f_{\mathrm{s}}$ is the modulating frequency. Thus, positive and negative half-wave asymmetry caused by the asynchronous modulation is small, and the lower order harmonics introduced can also be ignored. In the synchronous modulation region, the ratio $N$ remains constant. And near the base frequency of the traction motor, the three-level inverter needs to be operated in square-wave mode. The method of carrier disposition PWM [24] is widely used in the asynchronous modulation of three-level inverters; on the other hand, the 
accomplishment of square-wave is simple. Thus, the middle frequency of multi-mode PWM should be focused on.

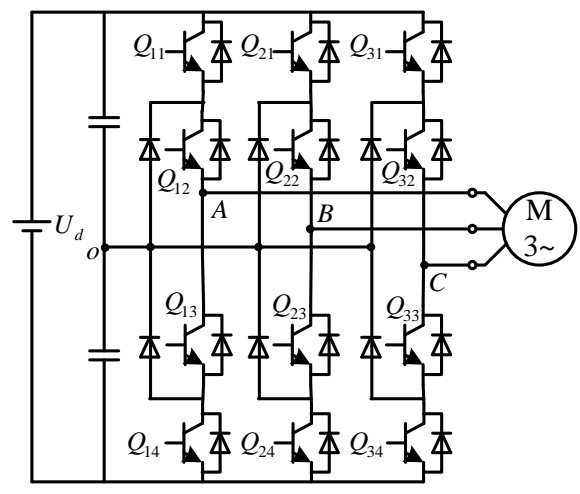

Figure 1. Three-level NPC Inverter Topology

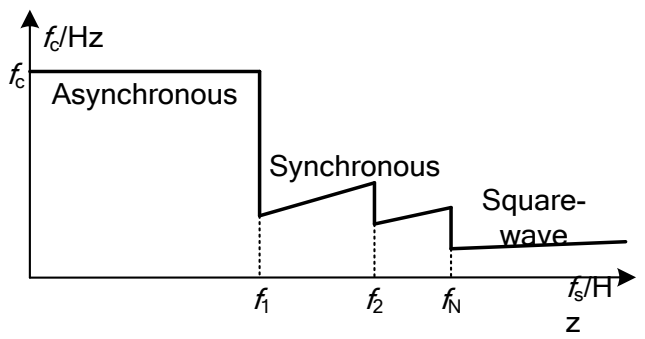

Figure 2. Transition Strategy of Multi-mode Modulation

\subsection{The Regular-sampled PWM at Low Carrier Frequency Ratio}

In the synchronous modulation stage, for the three-level inverter, the conventional regular-sampled PWM can't be used, otherwise the number of sampling points will reduce as the ratio $N$ goes down, which will lead to the situation that output fundamental voltage can't reach the voltage command when the carrier frequency ratio $N$ is 7,5, etc. And Figure 3 shows the output PWM pulse is obtained by regular-sampled PWM.

Figure 3(a) shows that the modulating wave is sampled at each peak of the carrier wave. Figure 3(b) shows that the sampled modulating wave is compared to the carrier wave. The PWM pulse pattern is obtained by the comparison between the two waves, as shown in Figure 3(c).

Assuming that the command amplitude $U_{1 \mathrm{~s}}$ of modulating wave in one cycle remains constant and the fundamental frequency is $\omega_{1}$ in $\mathrm{rad} / \mathrm{s}$ (same as the modulating frequency $f \mathrm{~s})$, the modulating wave can be given as

$$
u(t)=U_{1 s} \sin \left(\omega_{1} t\right)
$$

Then, the sampled modulating amplitude will be $U_{1 \mathrm{~s}}{ }^{*} \sin (\pi / 5)$ in the first carrier cycle. Assuming that the amplitude of the carrier wave is $U_{\mathrm{d}} / 2$, the angles $\theta_{1}$ and $\theta_{2}$ corresponding to the on-off action in Figure 3(b) can be calculated as

$$
\theta_{1}=\frac{U_{1 s} \sin (\pi / 5)}{U_{d} / 2} \cdot \frac{\pi}{5}
$$




$$
\theta_{1}=\frac{U_{1 s} \sin (\pi / 5)}{U_{d} / 2} \cdot \frac{\pi}{5}
$$

The angles $\theta_{3}$ and $\theta_{4}$ in Figure 3(b) can also be calculated in the same way as

$$
\begin{gathered}
\theta_{3}=\frac{U_{1 s} \sin (3 \pi / 5)}{U_{d} / 2} \cdot \frac{\pi}{5}+\frac{2 \pi}{5} \\
\theta_{4}=\frac{4 \pi}{5}-\frac{U_{1 s} \sin (3 \pi / 5)}{U_{d} / 2} \cdot \frac{\pi}{5}
\end{gathered}
$$

Thus, the Fourier series of the wave $u_{\mathrm{o}}$ in Figure 3(c) can be given as

$$
\begin{gathered}
u_{o}(t)=\sum_{n=1}^{\infty}\left[b_{n} \sin \left(n \omega_{1} t\right)\right] \\
b_{n}=\frac{2}{\pi} \int_{0}^{\pi} u_{o}(t) \sin \left(n \omega_{1} t\right) d\left(\omega_{1} t\right)
\end{gathered}
$$

where $b_{\mathrm{n}}$ is $\mathrm{n}^{\text {th }}$ harmonic amplitude and $b_{1}$ is fundamental amplitude.

Based on the above equations, the fundamental amplitude $b_{1}$ can be written from Equation (2) - (7) as

$$
\begin{aligned}
b_{1} & =\frac{2}{\pi}\left[\int_{0}^{\theta_{1}} \frac{U_{d}}{2} \sin \left(\omega_{1} t\right) d\left(\omega_{1} t\right)+\int_{\theta_{2}}^{\theta_{3}} \frac{U_{d}}{2} \sin \left(\omega_{1} t\right) d\left(\omega_{1} t\right)+\int_{\theta_{4}}^{4 \pi / 5} \frac{U_{d}}{2} \sin \left(\omega_{1} t\right) d\left(\omega_{1} t\right)\right] \\
& =\frac{U_{d}}{\pi}\left[1-\cos \theta_{1}+\cos \theta_{2}-\cos \theta_{3}+\cos \theta_{4}-\cos \left(\frac{4}{5} \pi\right)\right]
\end{aligned}
$$

Assuming that modulating depth $m$ is $U_{1 \mathrm{~s}} /\left(U_{\mathrm{d}} / 2\right)$ and the output fundamental voltage $u_{1 \mathrm{~s}}$ is $b_{1}$, then the voltage $u_{1 \mathrm{~s}}$ can be written as

$$
\begin{aligned}
u_{1 s}=\frac{U_{d}}{\pi} & {\left[1-\cos \theta_{1}+\cos \theta_{2}-\cos \theta_{3}+\cos \theta_{4}-\cos \left(\frac{4}{5} \pi\right)\right] } \\
=\frac{U_{d}}{\pi} & {\left[1-\cos \left(m \cdot \frac{\pi}{5} \sin \left(\frac{\pi}{5}\right)\right)+\cos \left(\frac{2 \pi}{5}-m \cdot \frac{\pi}{5} \sin \left(\frac{\pi}{5}\right)\right)-\cos \left(\frac{2 \pi}{5}+m \cdot \frac{\pi}{5} \sin \left(\frac{3 \pi}{5}\right)\right)+\right.} \\
& \left.\quad \cos \left(\frac{4 \pi}{5}-m \cdot \frac{\pi}{5} \sin \left(\frac{3 \pi}{5}\right)\right)-\cos \left(\frac{4 \pi}{5}\right)\right]
\end{aligned}
$$

Assuming that $u_{1 \mathrm{~s}}{ }^{*}$ is the output fundamental voltage of per unit, and $u_{1 \mathrm{~s}}{ }^{*}$ is $u_{1 \mathrm{~s}} / U_{1 \mathrm{~s}}$, then $u_{1 \mathrm{~s}}{ }^{*}$ can be written as

Figure 4 shows the variation of output fundamental voltage $u_{1 \mathrm{~s}}{ }^{*}$ with a variation of modulating depth $m$. 


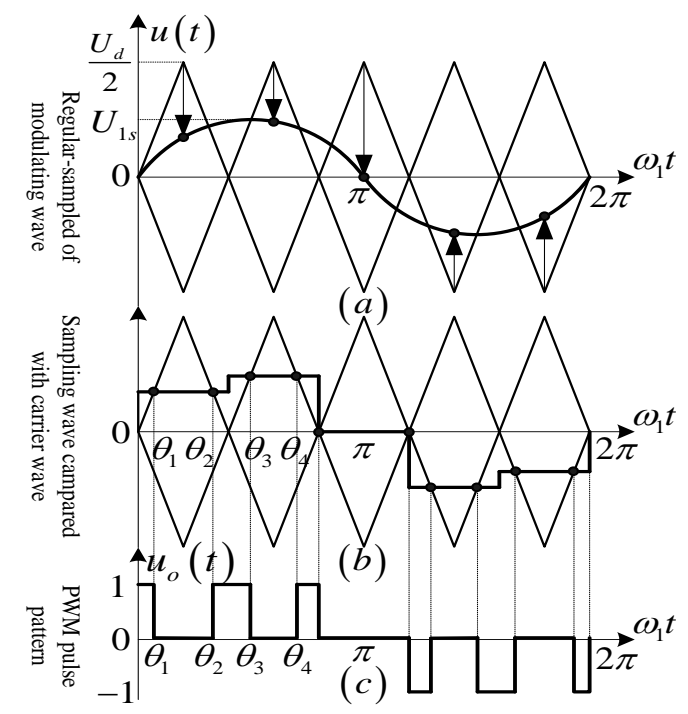

Figure 3. Regular-sampled PWM for $\mathrm{N}=5$

As shown in Figure 4, when the carrier frequency ratio $N$ is 5, the output fundamental voltage cannot reach the command voltage. In the same way, when the carrier frequency ratio $N$ is 7 and 3, the regular-sampled PWM cannot be used, or it will make it difficult to transform the modulation mode to square wave.

On the other hand, for the three-phase system, it may show asymmetry when the carrier frequency ratio $N$ is 7 or 5 , which are not multiple of 3 .

In this paper, a central $60^{\circ}$ synchronous modulation is used in the stage of synchronous modulation. And with this method, the output waveform can be obtained by calculating switching angles directly based on the command values rather than sampling method.

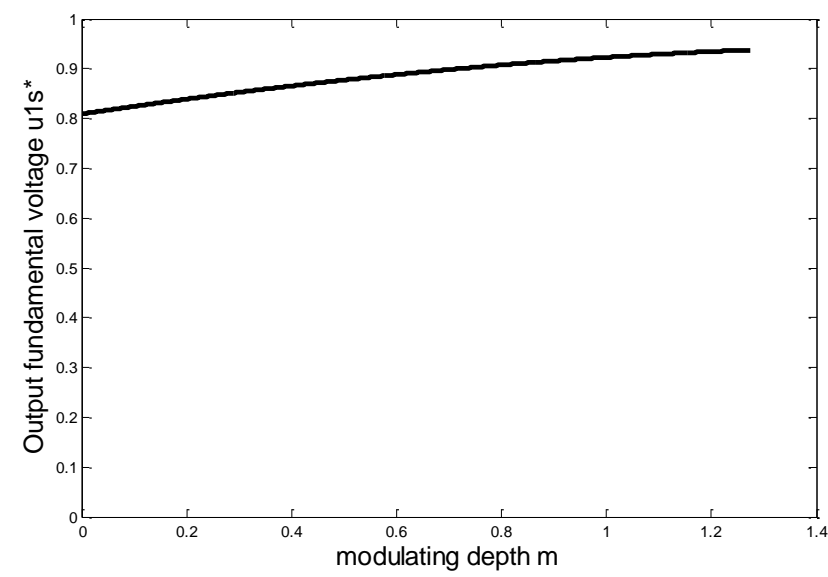

Figure 4. Variation of Output Fundamental Voltage $u_{1 s}{ }^{*}$ with Varying Modulating depth $\boldsymbol{m}$

\subsection{Principles of Central $60^{\circ}$ Synchronous Modulation and the Calculations of Switching Angles}

In central $60^{\circ}$ synchronous modulation mode, the carrier frequency ratio $N$ could be 7, 5, and 3. In this paper, the case of $N=5$ will be focused on, principle and accomplishment of which will be specifically analyzed. For $N=7$ and 3, the output waveforms and calculations of switching angles will be described briefly. 
2.3.1. Central $60^{\circ}$ Synchronous Modulation for $\mathbf{N}=5$ : In Figure 1, only consider the operation of phase A. Figure 5 shows phase voltage $u_{\text {Ao }}$ waveform of central $60^{\circ}$ synchronous modulation for $N=5$ and the corresponding switching waves, where $T_{\mathrm{s}}$ is a period of modulating wave and $T_{\mathrm{c}}$ is a period of carrier wave.

The output waveform with central $60^{\circ}$ synchronous modulation can be obtained by comparing the special carrier wave and the modulating wave, as shown in Figure 5. And the method of comparison is similar to regular-sampled PWM. The switching angles $\beta_{1}$ and $\beta_{2}$ only distributed in the central $60^{\circ}$ of half-cycle of the modulating wave in Figure 5. If analog control is used in the system, the output PWM pulses can be correctly obtained by natural-sampled based on the comparison method above. However, digital control is often used in practically for the high-power three-level inverter and the natural-sampled PWM is no longer applicable.

As shown in Figure 5, the special carrier wave and the modulating wave are all symmetrical about $\pi / 2$, so the switching angles $\beta_{1}$ and $\beta_{2}$ are symmetrical about $\pi / 2$ and they are equal to be $\beta$ here. The output waveforms $u_{\mathrm{Ao}}$ of central $60^{\circ}$ synchronous modulation has features of half-wave odd symmetry and quarter-wave symmetry. Assuming that $\beta$ is symmetrical about central line of the corresponding carrier wave. Thus, the Fourier series of the wave $u_{\mathrm{Ao}}$ can be given as

$$
\begin{gathered}
u_{A o}=\sum_{n=1}^{\infty}\left[b_{n} \sin \left(n \omega_{1} t\right)\right] \\
b_{n}=\frac{4}{\pi} \int_{0}^{\frac{\pi}{2}} u_{A o}(t) \sin \left(n \omega_{1} t\right) d\left(\omega_{1} t\right)
\end{gathered}
$$

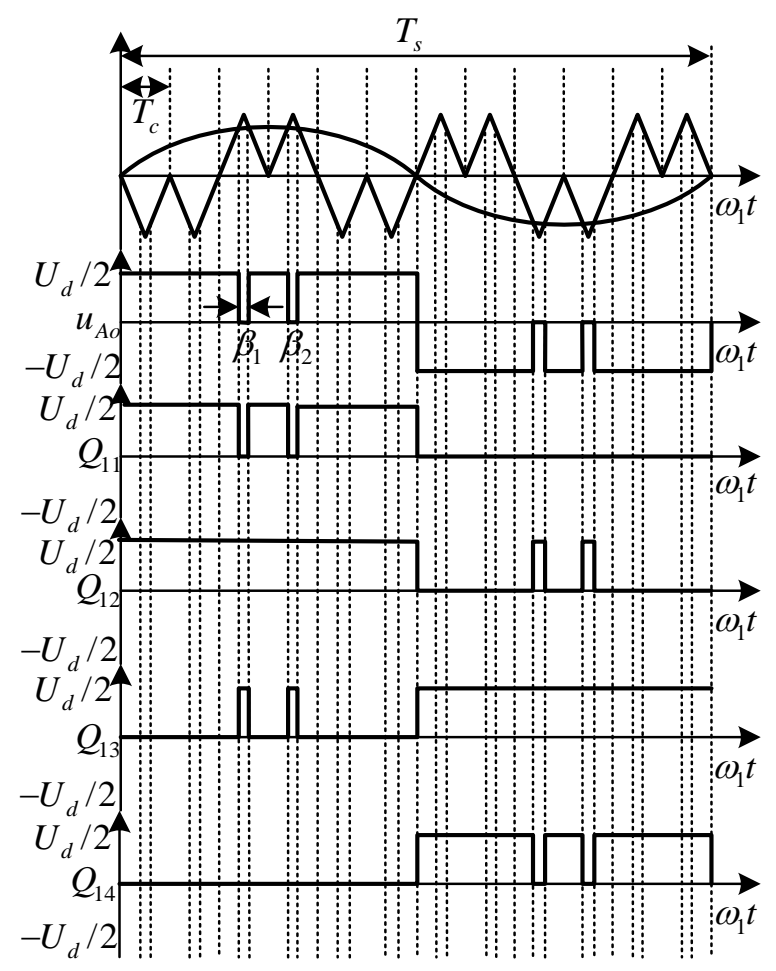

Figure 5. Phase Voltage Wave of Central $60^{\circ}$ Synchronous Modulation and the Corresponding Switching Waves 
Based on the assumption above, the equation of fundamental amplitude can be written from Equation (11-12) as

$$
\begin{aligned}
b_{1}= & \frac{4}{\pi}\left[\int_{0}^{\frac{5 \pi}{12}-\frac{\beta}{2}} \frac{U_{d}}{2} \sin \left(\omega_{1} t\right) d\left(\omega_{1} t\right)+\right. \\
& \left.\int_{\frac{5 \pi}{12}+\frac{\beta}{2}}^{\frac{\pi}{2}} \frac{U_{d}}{2} \sin \left(\omega_{1} t\right) d\left(\omega_{1} t\right)\right] \\
= & \frac{2 U_{d}}{\pi}\left[1-\frac{\sqrt{2}+\sqrt{6}}{2} \sin \frac{\beta}{2}\right]
\end{aligned}
$$

Assuming that the fundamental command is $U_{1 \mathrm{~s}}, \beta$ can be expanded as

$$
\beta=2 \arcsin \left(\frac{1-\pi U_{1 s} /\left(2 U_{d}\right)}{(\sqrt{2}+\sqrt{6}) / 2}\right)
$$

Then the output waveform $u_{\mathrm{Ao}}$ in the whole cycle can be determined by the obtained switching angle $\beta$.

2.3.2. Central $60^{\circ}$ Synchronous Modulation for $\mathbf{N}=7$ : In Figure 1, only consider the operation of phase A. Figure 6 shows phase voltage $u_{\mathrm{Ao}}$ waveform of central $60^{\circ}$ synchronous modulation for $N=7$.

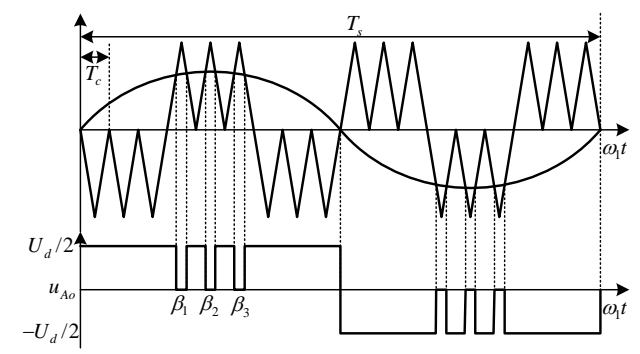

Figure 6. Central $60^{\circ}$ Synchronous Modulation for $\mathrm{N}=7$

Note that in Figure $6, \beta_{1}, \beta_{2}$ and $\beta_{3}$ are switching angles in half-cycle of the modulating wave. Assuming that $\beta_{1}=\beta_{2}=\beta_{3}$, in order to simplify the calculation, and each of them are symmetrical about the central line of their corresponding carrier wave, the Fourier series of the wave $u_{\mathrm{Ao}}$ can be given as

$$
\begin{aligned}
b_{1} & =\frac{4}{\pi}\left[\int_{0}^{\frac{7 \pi}{18}-\frac{\beta}{2}} \frac{U_{d}}{2} \sin \left(\omega_{1} t\right) d\left(\omega_{1} t\right)+\right. \\
& \left.\int_{\frac{7 \pi}{18}+\frac{\beta}{2}}^{\frac{\pi}{2}-\frac{\beta}{2}} \frac{U_{d}}{2} \sin \left(\omega_{1} t\right) d\left(\omega_{1} t\right)\right] \\
& =\frac{2 U_{d}}{\pi}\left[1-\left(2 \sin \frac{7 \pi}{18}+1\right) \sin \frac{\beta}{2}\right] \\
& =\frac{2 U_{d}}{\pi}\left(1-2.8794 \sin \frac{\beta}{2}\right)
\end{aligned}
$$

Assuming that the fundamental command is $U_{1 \mathrm{~s}}, \beta$ can be expanded as 


$$
\beta=2 \arcsin \left(\frac{1-\pi U_{1 s} /\left(2 U_{d}\right)}{2.8794}\right)
$$

\section{Accomplishment of Central $60^{\circ}$ Synchronous Modulation for Three-level Inverters}

Only consider $N=5$ for central $60^{\circ}$ synchronous modulation, switching angle $\beta$ can be calculated based on Equation (14) for the specific fundamental amplitude $U_{1 \mathrm{~s}}$. As indicated in Figure 5, when the switching angle $\beta$ is calculated at the starting point of each modulating cycle, the output voltage of the whole cycle for phase A can be determined. The output phase voltage $u_{\mathrm{Ao}}$ will be like the pattern shown in Figure 5.

As shown in Figure 5, based on the switching angle $\beta$, the expected amplitude of output waveform $u_{\mathrm{Ao}}$ at each phase position can be determined. As indicated in Figure 3, only consider quarter-cycle of the output waveform, switching angle is symmetrical about $5 \pi / 12$. Then, using the carrier phase as reference, the expected amplitude of output waveform $u_{\mathrm{Ao}}$ is $U_{\mathrm{d}} / 2$ when the modulating phase is greater than or equal to 0 and less than $5 \pi / 12-\beta / 2$, and it's 0 when the modulating phase is greater than or equal to $5 \pi / 12-\beta / 2$ and less than $5 \pi / 12+\beta / 2$. When the modulating phase is greater than or equal to $5 \pi / 12+\beta / 2$ and less than $\pi / 2$, it will be $U_{\mathrm{d}} / 2$ again. The expected amplitude of the rest three-quarter-cycle of output waveform $u_{\mathrm{Ao}}$ can be written in the same way.

When realizing the output of $u_{\mathrm{A}}$, the actual modulating phase $\varphi$ should be compared with phase of the switching on-off point, such as $5 \pi / 12-\beta / 2,5 \pi / 12+\beta / 2$, and so on, where $\varphi$ can be calculated as $\varphi=\int 2 \pi f_{s} d t$. And the specific details of comparison method, for the quarter-cycle of output waveform $u_{\mathrm{Ao}}$ is shown in Figure 7, the chat is flow of obtaining output phase voltage $u_{\mathrm{Ao}}$ waveform.

As shown in Figure 7, the output phase voltage $u_{\mathrm{Ao}}$ of each entire cycle can be obtained by the calculation of switching angle $\beta$. Then, based on the relationship between phase A, phase $\mathrm{B}$, and phase $\mathrm{C}$, the output voltage of phase $\mathrm{B} u_{\mathrm{Bo}}$ and phase $\mathrm{C} u_{\mathrm{Co}}$ can be deduced. Figure 8 shows output voltage of three phases in one cycle.

Figure 8 shows that the determined three-phase voltage for inverter in one cycle. According to the principle of three-level inverters operation, only consider the operation of the bridge of phase A, current will be carried by devices $Q_{11}$ and $Q_{12}$ when $u_{\mathrm{Ao}}$ is $U_{\mathrm{d}} / 2$, by devices $Q_{12}$ and $Q_{13}$ when $u_{\mathrm{Ao}}$ is 0 and which clamp the output potential to the neutral point with the help of clamping diodes, and by devices $Q_{13}$ and $Q_{14}$ when $u_{\mathrm{Ao}}$ is $-U_{\mathrm{d}} / 2$. Therefore, the corresponding switching waveform of each device can be determined, as indicated in Figure 5, and they can also be determined for the bridge of phase B and C in the same way. 


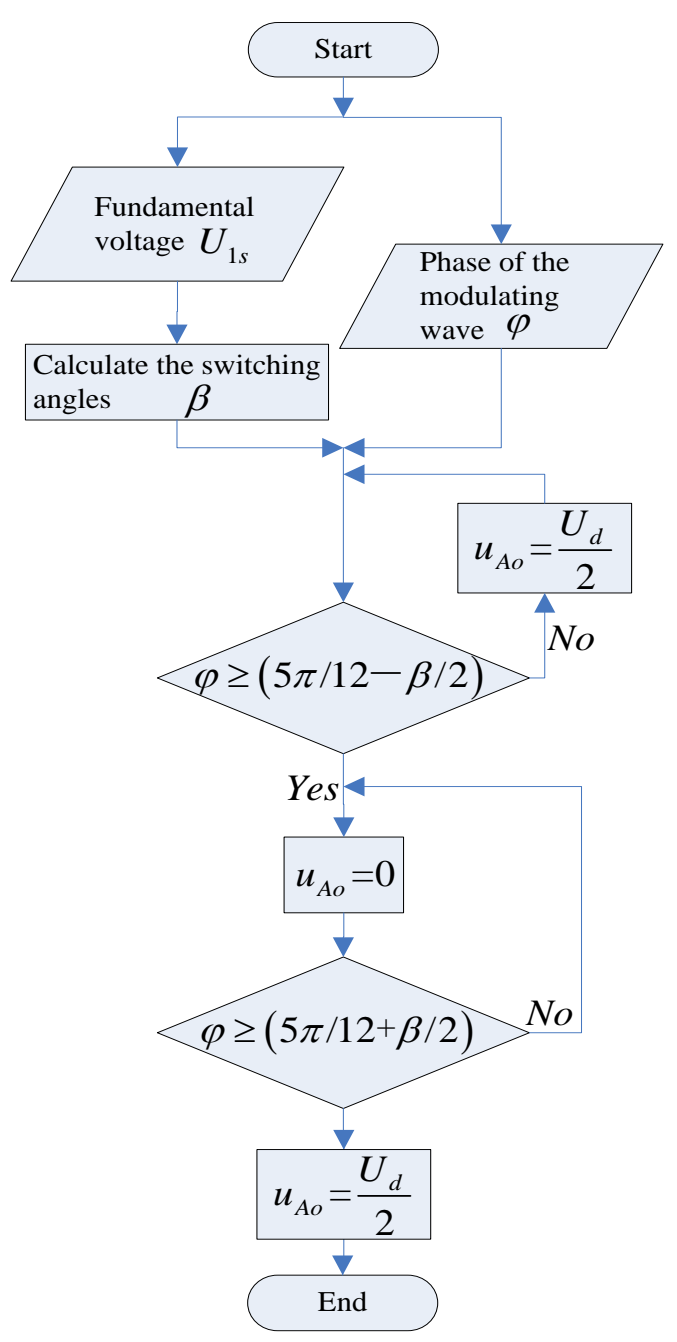

Figure 7. Flow Chart for $\mathrm{N}=5$

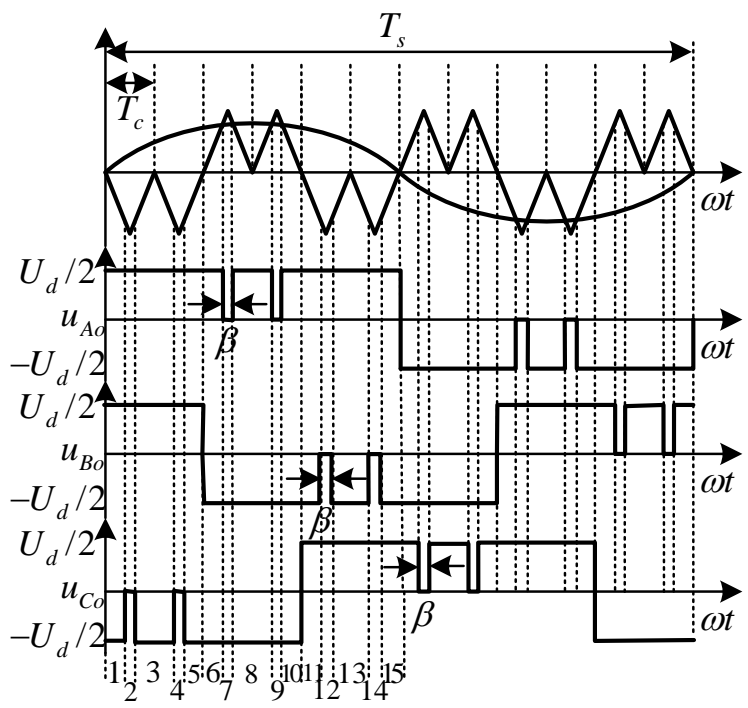

Figure 8. Output Phase Voltages for Three-level Inverter 
As indicated in Figure 8, only consider first half-cycle of the modulating wave, there are 15 switching states. Table 1 gives the summary of the switching states. Among them, state 1, 3, and 5 correspond to the circuit in Figure 9(a), and phase A and B are connected to the positive bus and phase $\mathrm{C}$ is connected to the negative bus. State 2 and 4 correspond to circuit in Figure 9(b), and phase A and B are connected to the positive bus and phase A is clamped to the neutral point. State 6, 8, and 10 correspond to the circuit in Figure 9(c), state 7 and 9 correspond to the circuit in Figure 9(d), state 11, 13, and 15 correspond to the circuit in Figure 9(e), and state 12 and 14 correspond to the circuit in Figure 9(f) .

Table 1. Summary of Inverter Switching States

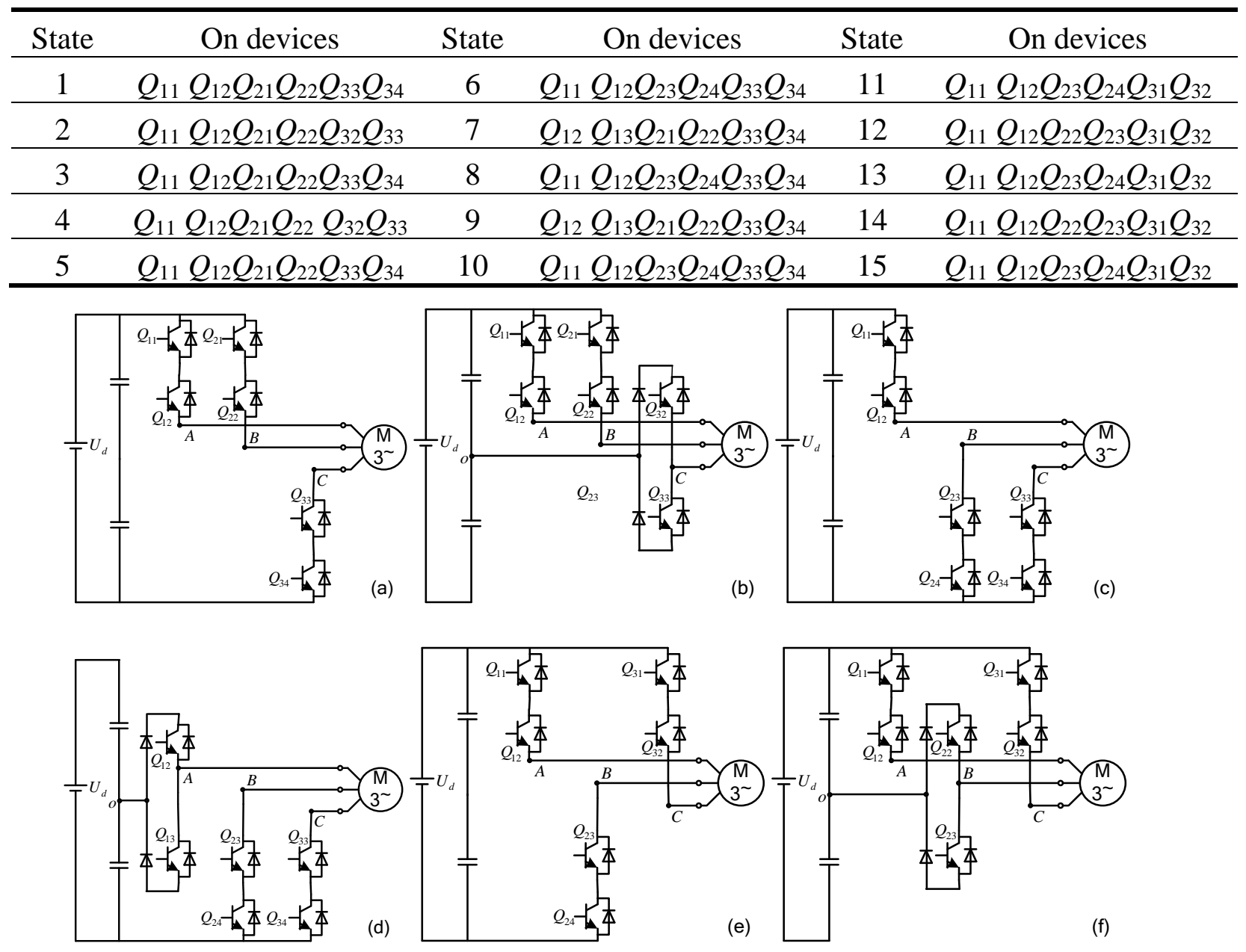

Figure 9. Switching States of Central $60^{\circ}$ Synchronous Modulation for $\mathrm{N}=5$

\section{Performance Evaluation by Simulation}

A drive system with three-level inverter and induction motor is simulated by Matlab/Simulink in this paper, where the rated power of motor is $365 \mathrm{~kW}$, rated voltage is $2000 \mathrm{~V}$, rated frequency is $140 \mathrm{~Hz}$, and the DC-link voltage is $3000 \mathrm{~V}$. The modulation modes used in the simulation are asynchronous modulation, then central $60^{\circ}$ synchronous modulation for $N=5$, followed by central $60^{\circ}$ synchronous modulation for $N=3$, then square-wave. The carrier frequency of asynchronous modulation is $1000 \mathrm{~Hz}$. The modulation mode will switching from asynchronous modulation to central $60^{\circ}$ synchronous modulation for $N=5$ when the modulating frequency is $58 \mathrm{~Hz}$, switching to 
central $60^{\circ}$ synchronous modulation for $N=3$ when the modulating frequency is $90 \mathrm{~Hz}$, and switching to square-wave when the modulating frequency is $140 \mathrm{~Hz}$, as shown in Figure 2.

Figure 10 shows the switching angles for different modulation modes. Note that the fundamental voltage command increases as the modulating frequency rises up in the region below the base frequency, remains the maximum voltage in the region above the base frequency, the switching angles need to be calculated and the values will decrease as the modulating frequency rises up in the region of central $60^{\circ}$ synchronous modulation, and the switching angle is 0 when the inverter operates in square-wave mode.

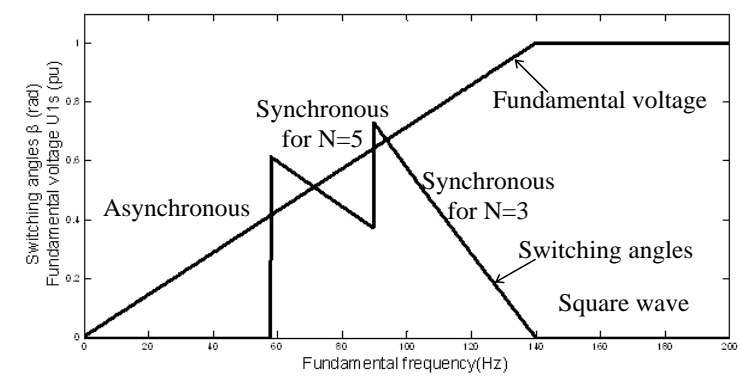

\section{Figure 10. Switching Angles for Different Modulation Modes}

Figure 11 shows the simulation results of switching between different modulation modes, line voltage and current, in this order, are shown. The results indicate that smooth switching with low impulse current among different modes is accomplished by using the above switching strategy. And the output waveform can smoothly switching from central $60^{\circ}$ synchronous modulation for $N=3$ to square wave, as shown in Figure 10 and 11(c).

Figure 12 shows the spectrum analysis of central $60^{\circ}$ synchronous modulation. The results indicate that the even order and multiple of 3 order harmonics are eliminated by using central $60^{\circ}$ synchronous modulation. And the simulation results are consistent with the theoretical analysis.

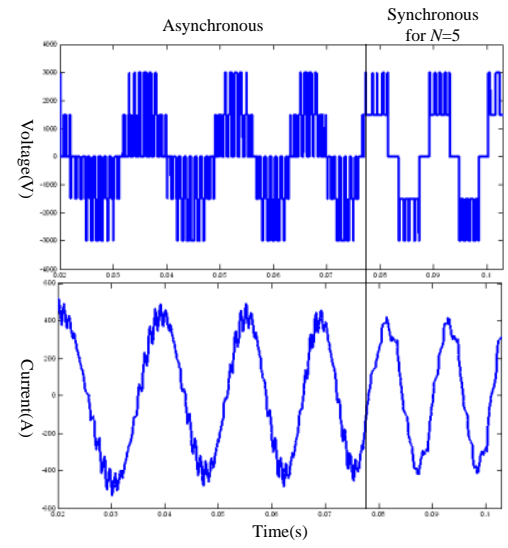

(a) Switching from Asynchronous Modulation to Central $60^{\circ}$ Synchronous Modulation for $\mathrm{N}=5$ 


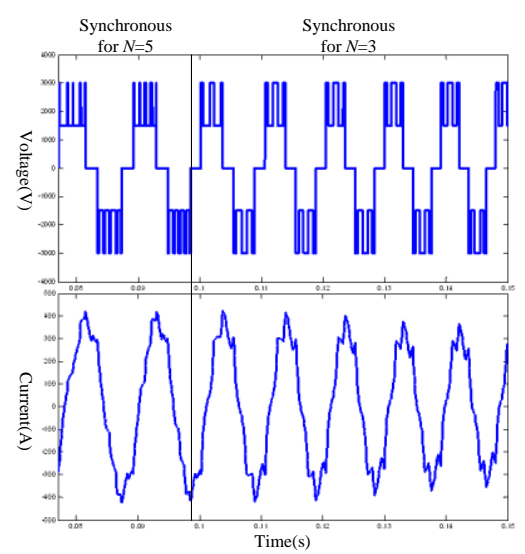

(b) Switching from Central $60^{\circ}$ Synchronous Modulation for $\mathrm{N}=5$ to $\mathrm{N}=3$

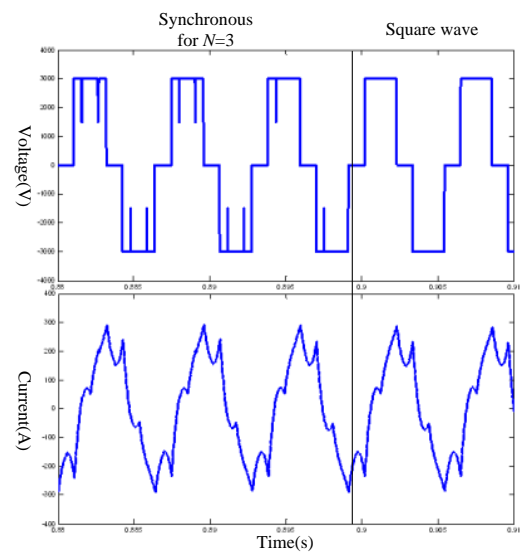

(c) Switching from Central $60^{\circ}$ Synchronous Modulation for $\mathrm{N}=3$ to Square Wave

Figure 11. Transition between Different Modulation Modes

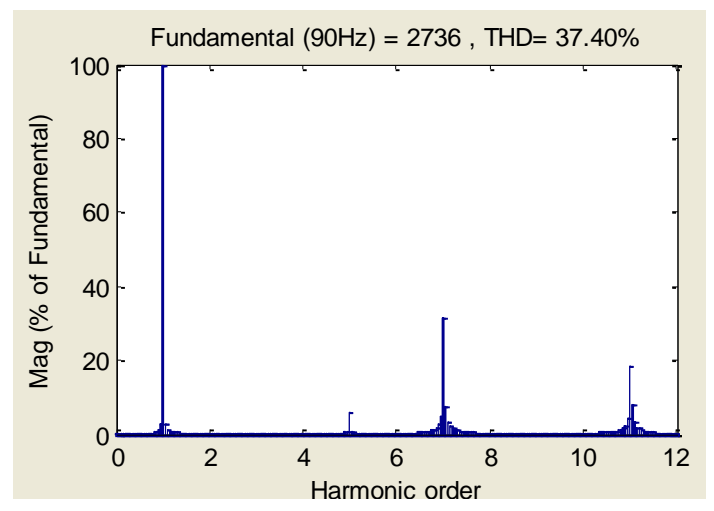

Figure 12. Spectrum Snalysis of Central $60^{\circ}$ Synchronous Modulation

Assuming that regular-sampled PWM and central $60^{\circ}$ synchronous modulation for $N=5$ are respectively used in the modulation frequency region ranging from $80 \mathrm{~Hz}$ to $90 \mathrm{~Hz}$, Figure 13 shows the corresponding curves of the phase voltage harmonics and Figure 14 shows the fundamental output voltage under the two modulation modes. Figure 13 indicates that the even order and the multiple of 3 order harmonics are eliminated under the central $60^{\circ}$ synchronous modulation. Compared with the regular-sampled PWM, the output waveform under the central $60^{\circ}$ synchronous modulation has a better symmetry 
between the three phase of the motor. Figure 14 indicates that the output fundamental voltage under regular-sampled modulation cannot reach the command voltage gradually as the modulating frequency rises.
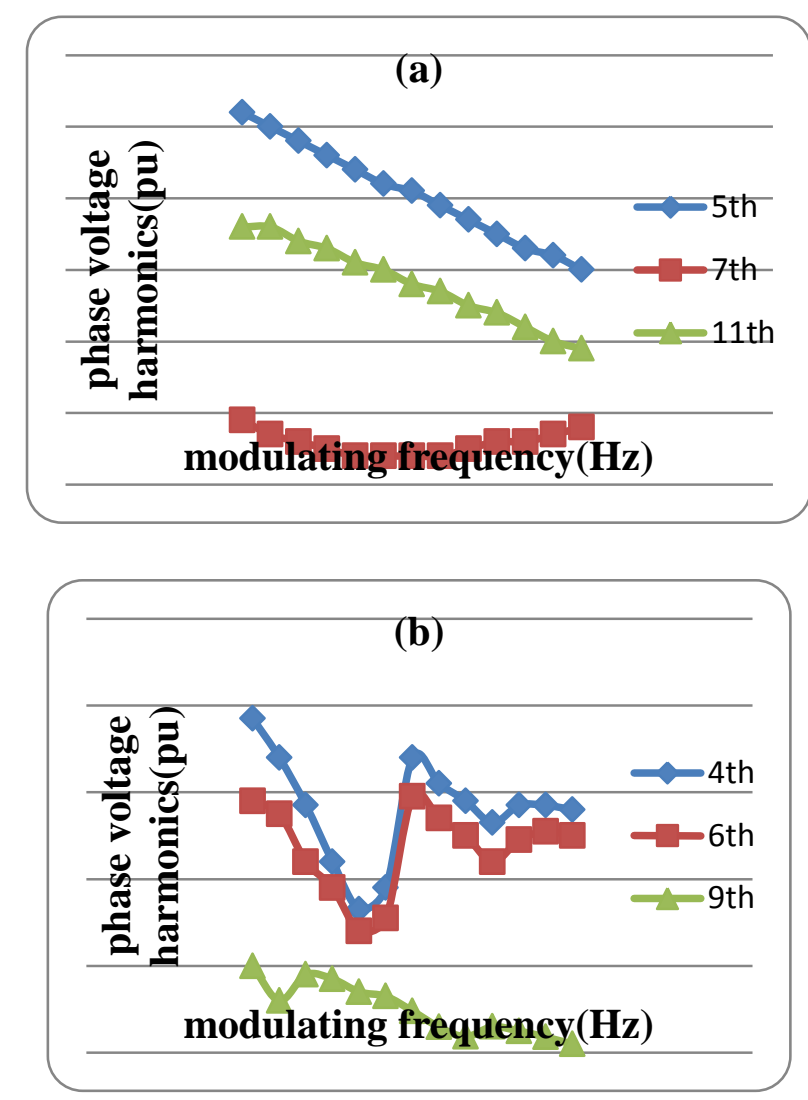

(a) Central $60^{\circ}$ Synchronous Modulation for $\mathrm{N}=5$; (b) Regular-sampled Modulation for $\mathrm{N}=5$

Figure 13. Output Phase Voltage Harmonics under Different Modulation Modes

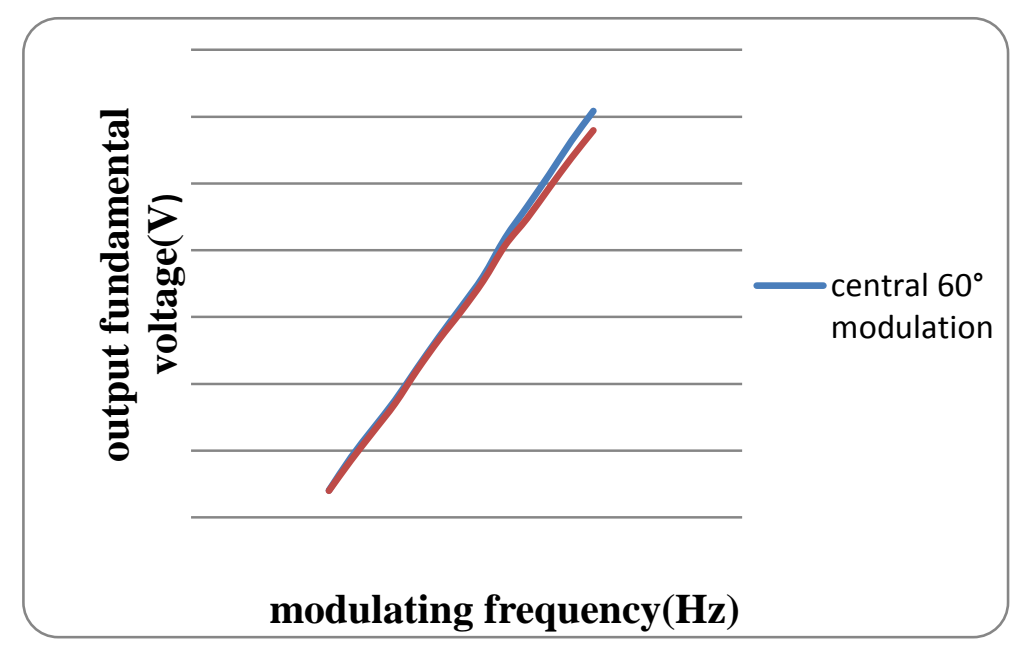

Figure 14. Output Fundamental Voltage under Different Modulation Modes 


\section{Conclusions}

The multi-mode PWM for diode clamped three-level inverters are researched in this paper, especially the central $60^{\circ}$ synchronous modulation is analyzed in detail. The output waveform of the central $60^{\circ}$ synchronous modulation has a good symmetry and the even order harmonic is eliminated. The multiple of 3 order harmonics are eliminated even when the carrier frequency is not multiple of 3 . And, the switching angle is calculated online, which can improve the dynamic performance of the system. The smooth switching among different modes with low impulse current is also accomplished, which can improve the reliability of the system.

\section{Acknowledgments}

This work was supported by a grant from the Major State Basic Research Development Program of China (973 Program: 2011CB711100).

\section{References}

[1] R. Teichmann and S. Bernet, "A comparison of three-level converters versus two-level converters for low-voltage drives, traction, and utility application”, IEEE Transaction on Industry Applications, vol. 41, no. 3, (2005), pp.855-865.

[2] W. Jing, G. J. Tan, Z. B. Ye, "Comparison of two-level and three-level converters in permanent magnet direct-drive wind power generation system", Automation of Electric Power Systems, vol. 35, no.6, (2011), pp.92-97.

[3] V. T. Ranganathan, "Modified SVPWM algorithm for three level VSI with synchronized and symmetrical waveform", IEEE Transactions on Industrial Electronics, vol.54, no.1, (2007), pp. 486-494.

[4] A. K. Rathore, J. Holtz, and T. Boller, "Synchronous optimal pulse width modulation for low-switching-frequency control of medium-voltage multilevel inverters", IEEE Transactions on Industrial Electronics, vol.57, no.7, (2010), pp. 2374-2381.

[5] T. Boller, J. Holtz and A. K. Rathore, "Neutral point potential balancing using synchronous optimal pulsewidth modulation of multilevel inverters in medium voltage high power AC drives", Energy Conversion Congress and Exposition (ECCE), (2012) September 15-20; Raleigh, NC, USA.

[6] A. K. Rathore, J. Holtz and T. Boller, "Optimal pulse width modulation of multilevel inverters for low switching frequency control of medium voltage high power industrial AC drives", Energy conversion congress and exposition, IEEE, (2010) September 12-16; Atlanta,USA.

[7] K. Lee, G. Nojima, "Quantitative power quality and characteristic analysis of multilevel pulse width modulation methods in medium voltage high power industrial drives", Energy conversion congress and exposition, IEEE, (2011) September 17-22 ; Phoenix, USA.

[8] J. Lago, M. L. Heldwein, "Multilevel synchronous optimal pulse width modulation generalized formulation", Control and Modeling for Power Electronics (COMPEL), (2014) June22-25; Santander, Spain.

[9] G. Narayanan, V. T. Ranganathan, "Extension of operation of space vector PWM strategies with low switching frequencies using different over modulation algorithms", IEEE Transactions on Power Electronics, vol.17, no.5, (2002), pp.788-798.

[10] M. Y. Ye, B. Li and S. Li, "Study on walsh function based multi-band SHEPWM control technology for multi-level inverter", Power System Technology, vol.37, no.10, (2013), pp.2945-2952.

[11] W. M. Fei, X. B. Ruan and B. Wu, "A generalized formulation of quarter-wave symmetry SHE-PWM problems for multilevel inverters", IEEE Transactions on Power Electronics, vol. 24, no.7, (2009), pp.1758 - 1766 .

[12] W. M. Fei, X. B. Ruan, and B. Wu, "A generalized half-wave symmetry SHE-PWM formulation for multilevel voltage inverters", IEEE Transactions on Industrial Electronics, vol. 57, no. 9, (2010), pp. 3030-3038.

[13] K. L. Zhou, Y. H. Yang, F. Blaabjerg, W. Z. Lu and D. W. Wang, "Selective harmonic control for power converters", Energy Conversion Congress and Exposition (ECCE), (2014) September14-18; Pittsburgh, PA, USA.

[14] J. Napoles, J. I. Leon and R. Portillo, "Selective harmonics mitigation technique for high-power converters”, IEEE Transactions on Industrial Electronics, vol.57, no.7, (2010), pp.2315-2323. 
[15] S. R. Bowes, "Optimal Regular-sampled PWM Inverter control techniques", IEEE Transactions on Industrial Electronics, vol.54, no.3, (2007), pp.1547-1559.

[16] B. K. Bose, "Modern Power Electronics and AC Drives", San Antonio: Pearson Education, (2002).

[17] M. Kiran kumar, A. Madhu Sainath and V. Pavan Kumar, "Selective harmonic elimination by programmable pulse width modulation in inverters", International Journal of Engineering Trends \& Technology, vol.4, no.4, (2013), pp.1547-1559.

[18] R. S. Wang, H. G. Wu, "Numerical analysis of harmonic influence for selected harmonic elimination PWM", Transactions of China Electrotechnical Society, vol.26, no.9, (2011), pp.183-189.

[19] J. Holtz, N. Oikonomou, "Fast dynamic control of medium voltage drives operating at very low switching frequency-an overview", IEEE Transactions on Industrial Electronics, vol.55, no.3, (2008), pp.1005-1013,

[20] J. Holtz, X. Qi, "Optimal control of medium-voltage drives-an overview", IEEE Transactions on Industrial Electronics, vol.60, no.12,(2013), pp.5472-5481.

[21] N. Oikonomou, C. Gutscher, P. Karamanakos, D. K. Frederick and G. Tobias, "Model predictive pulse pattern control for the five-level active neutral-point-clamped inverter", Industry Applications IEEE Transactions on, vol.49, no.6, (2013), pp.2583-2592.

[22] N. Oikonomou, J. Holtz, "Closed-loop control of medium voltage drives operated with synchronous optimal pulse width modulation”, IEEE Transactions on Industry Applications, vol.44, no.1, (2008), pp.115-123.

[23] J. Holtz, N. Oikonomou, "Synchronous optimal pulse width modulation and stator flux trajectory control for medium-voltage drives", IEEE Transactions on Industry Applications, vol.43, no.2, (2007), pp.600-608.

[24] D. G. Holmes, A. T. Lipo, "Pulse width modulation for power converters: principle and practice", New York: Wiley-IEEE Press, (2003).
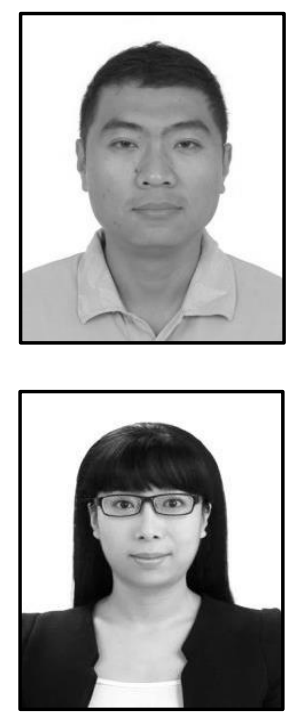

\section{Authors}

Fei Lin, He was born in Shandong Province, China in 1975. He received his B.S., M.S. and Ph.D. in Electrical Engineering from Xi'an Jiaotong University, China, Shandong University, China and Tsinghua University,China, in 1997, 2000 and 2004, respectively. Since 2004, he has been with School of Electrical Engineering, Beijing Jiaotong University, where he is currently an Associate Professor. His research interests are in the areas of power electronics and motor control.

Qiaona Lian, She was born in Hebei Province, China in 1991. She received his B.S.in Electrical Engineering from Yanshan University, China, in 2013. Currently, she is a graduate student of Beijing Jiaotong University. Her research interests are in the areas of power electronics. 
International Journal of Smart Home

Vol. 9, No. 12, (2015) 